\title{
VELOCITY MEASUREMENTS OF TURBULENT WAKE FLOW OVER A CIRCULAR CYLINDER
}

\author{
CHANG-LUNG SHIH \\ Department of Aeronautics and Astronautics, National Cheng Kung University, 1, University Road \\ Tainan, 70101, Taiwan \\ p46024419@mail.ncku.edu.tw \\ WEI-CHENG CHEN \\ Department of Aeronautics and Astronautics, National Cheng Kung University, 1, University Road \\ Tainan, 70101, Taiwan \\ kb6kimo@hotmail.com \\ KEH-CHIN CHANG \\ Department of Aeronautics and Astronautics, National Cheng Kung University, 1, University Road \\ Tainan, 70101, Taiwan \\ kcchang@mail.ncku.edu.tw \\ MUH-RONG WANG \\ Department of Aeronautics and Astronautics, National Cheng Kung University, 1, University Road \\ Tainan, 70101, Taiwan \\ wangmr@mail.ncku.edu.tw
}

Published 22 June 2016

\begin{abstract}
There are two general concerns in the velocity measurements of turbulence. One is the temporal characteristics which governs the turbulent mixing process. Turbulence is rotational and is characterized by high levels of fluctuating vorticity. In order to obtain the information of vorticity dynamics, the spatial characteristics is the other concern. These varying needs can be satisfied by using a variety of diagnostic techniques such as invasive physical probes and non-invasive optical instruments. Probe techniques for the turbulent measurements are inherently simple and less expensive than optical methods. However, the presence of a physical probe may alter the flow field, and velocity measurements usually become questionable when probing recirculation zones. The non-invasive optical methods are mostly made of the foreign particles (or seeding) instead of the fluid flow and are, thus, of indirect method. The difference between the velocities of fluid and foreign particles is always an issue to be discussed particularly in the measurements of complicated turbulent flows. Velocity measurements of the turbulent wake flow over a circular cylinder will be made by using two invasive instruments, namely, a cross-type hot-wire anemometry (HWA) and a split-fiber hot-film anemometry (HFA), and a non-invasive optical instrument, namely, particle image velocimetry (PIV) in this study. Comparison results show that all three employed diagnostic techniques yield similar measurements in the mean velocity while somewhat deviated results in the root-mean-squared velocity, particularly for the PIV measurements. It is demonstrated that HFA possesses more capability than HWA in the flow measurements of wake flow. Wake width is determined in terms of either the flatness factor or
\end{abstract}

This is an Open Access article published by World Scientific Publishing Company. It is distributed under the terms of the Creative Commons Attribution 3.0 (CC-BY) License. Further distribution of this work is permitted, provided the original work is properly cited. 
shear-induced vorticity. It is demonstrated that flow data obtained with the three employed diagnostic techniques are capable of yielding accurate determination of wake width.

Keywords: Hot-wire anemometry; split-fiber hot-film anemometry; PIV; wake.

\section{Introduction}

Flow over circular cylinder (namely, wake) is a simple and useful model for many applications concerning obstacles to flow. Circular cylinder flow seems to be one of the simplest geometrics; however, it generates highly complex flow structures and has attracted a great deal of attention ${ }^{1}$. Flow over a circular cylinder has been studied extensively, both experimentally and numerically. The flow over a circular cylinder exhibits vastly different behaviors as the Reynolds number, based on the free-stream velocity, cylinder diameter, and kinematic viscosity, increases from zero to large values. There are a variety of diagnostic techniques which are in principle split into invasive/non-invasive approaches employed in the measurements of turbulent flows. In addition, there are two different analyses for turbulent flow quantities, namely, temporal and spatial correlations. Most of the intrusive diagnostics such as hot-wire anemometry (HWA) and hot-film anemometry (HFA) and some non-intrusive diagnostics such as laser Doppler velocimetry (LDV) are of point-wise measuring techniques which can provide the time-sequential data of instantaneous velocities at a fixed point with good temporal resolution. In contrast, some non-intrusive diagnostics such as particle image velocimetry (PIV), which is of simultaneous multi-point measuring technique, can provide the instantaneous velocities in a twodimensional domain with good spatial resolution so that the turbulent flow properties such as vorticity and the dissipation rate of turbulent kinetic energy can be calculated from the velocity distribution information with the accurate spatial differential quantities of turbulence.

In this study, the velocity measurements of turbulent flow over a long circular cylinder are made with a X-array hot-wire probe and a split-fiber hot-film probe, which are of the intrusive diagnostics and can provide the velocity data with good temporal resolution, as well as a PIV which is of the nonintrusive diagnostics and can provide the velocity data with good spatial resolution. Comparison of the measured mean velocity (first order statistics) and root-mean squared fluctuating velocity (second order statistics) using these three diagnostic techniques are made. The merits and defects of the three employed diagnostics on measurements of turbulent flow quantities are next discussed.

The characteristic length scale of wake is generally represented by the wake width, $l_{u}$, which is defined by $l_{u}=2 y_{\Delta}$ with the centerline position of the wake being set at $y=0$ in each section. Here the half-width $y_{\Delta}$ is defined as the transverse position of $\left(U-U_{c}\right) /\left(U_{\infty}\right.$ - $U_{c}$ ) being equal to $\Delta,(\Delta$ value is dependent on the accuracy in the determination of velocity). Here $U_{\infty}$ and $U_{c}$ are the mean stream-wise velocities in the free stream and at the centerline, respectively. However, it is argued ${ }^{1-3}$ that the phenomenon of intermittency, which is an evidence of turbulent fluctuations, in the outer region of the wake width, 
defined by $l_{u}$, can be still observed. Different ways to determine the interface between the inner shear turbulence and the outer free stream have been reported in the literature. For example, by analyzing the intermittency factor, which is estimated with the proportion of intermittent time by placing the sensor in the turbulent flow field, one can extract the interface of the activity of entrainment ${ }^{2}$. In addition, Lee and $\mathrm{Chang}^{4}$ determined the wake width by monitoring the sectional distributions of high-order turbulence statistics such as the flatness (Kurtosis) factor $\left(F_{u}\right)$ of the turbulent stream-wise velocity component which is defined as

$$
F_{u}=<u^{4}>/\left(u_{r m s}^{\prime}\right)^{4}
$$

where the lower and upper cases denote the instantaneous and mean quantities, respectively, while the superscript " ", the subscript " rms" and the symbol " $<>$ " denote the fluctuating quantity, root-mean-squared value and ensemble averaging operator, respectively. However, the two aforementioned definitions of wake width need a large number of raw data in the velocity measurements to achieve the stationary statistics of either the intermittency factor or the flatness factor around the edges (i.e., intermittency) of the shear turbulence regions.

In fact, turbulence is composed of eddies. Turbulence extracts energy from the mean flow in large eddies and this gain is approximately balanced by the viscous dissipation of energy in small scales. Furthermore, turbulence energy is transferred from large scales to small scales by vortex stretching processes. Therefore, it is the vorticity field, and not the velocity field, which is more fundamental to turbulence. However, the accurate evaluation of vorticity requires high spatial differential quantity of instantaneous velocity information, which cannot be obtained by using the point-wise measuring techniques. As a result, very few definitions of wake width were defined on the basis of vorticity field in the past. In addition, the conventional vortex-identification methods usually decompose the motion near a point into a pure irrational straining motion along the principal axes of the rate of the strain tensor (which generally includes a uniform dilatation) and a rigid-body rotation, so it is impossible to distinguish between the pure shearing motion and the actual swirling motion of a vortex. However, by using the triple decomposition method proposed by $\mathrm{Kolar}^{5}$, the total vorticity is split into two distinct parts : the shear component (shearinduced vorticity) and the residual component (rigid-body motion, which represents a direct measure of the actual swirling motion of a vortex). Chang and $\mathrm{Li}^{6}$ recently proposed a new definition of the characteristic length scale of the shear turbulence region in a mixing layer on the basis of the mean shear-induced vorticity field in which the measured data were obtained with a PIV.

The wake widths behind the circular cylinder are made with two alternative definitions in this study. One follows what was proposed by Lee and Chang ${ }^{4}$ and in terms of the flatness factor. The other follows what was proposed by Chang and $\mathrm{Li}^{6}$ and in terms of the shear-induced vorticity. 


\section{Experimental Aspects}

\subsection{Experimental facility}

The experimental facility used in this study is a vertically downward, rectangular, suction-type wind tunnel, schematically shown in Fig. 1., which is composed of a settling chamber, contraction, test section, and noise reduction chamber. This wind tunnel has a test section with a cross-sectional area of $150 \times 150 \mathrm{~mm}$ and length of $600 \mathrm{~mm}$, where the cylinder is mounted at the middle of the cross section, $75 \mathrm{~mm}$ down from the top of the test section. A Cartesian coordinate is set such that the transverse coordinate $y$ is positive toward the right and the stream-wise coordinate $\mathrm{x}$ is positive downward with the origin at the center of the cylinder. The diameter of the cylinder, $\mathrm{d}$, is $10 \mathrm{~mm}$ and the slenderness ratio of the cylinder is equal to 15 which were reported $^{4}$ to be capable of yielding wake flow with two-dimensional characteristics in the central span-wise regions of the domain of interest.

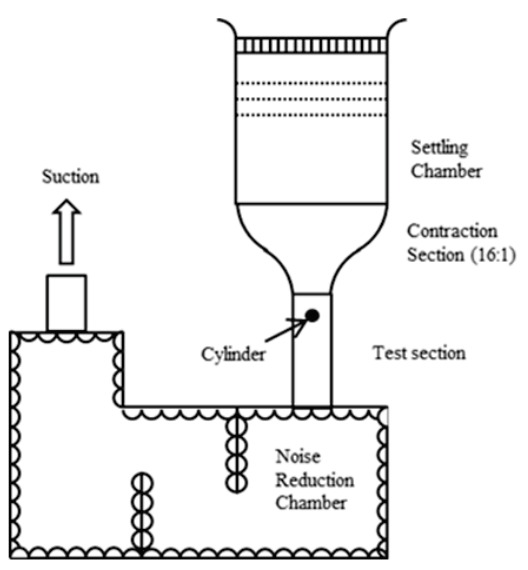

Fig. 1. Schematics of the experimental set-up

\subsection{Measurement instrumentation}

Two-component velocity measurements are made with the hot-wire/film anemometry (HWA/HFA, the StreamLine Pro System of Dantec Dynamics Inc.) in which each is composed of a mainframe (90N10) with a temperature probe included and the two modules of a constant temperature anemometer (CTA, 90C10). The X-array hot-wire probe has two $5 \mu \mathrm{m}$ platinum-plated tungsten sensing elements, which are about $1.25 \mathrm{~mm}$ long and are positioned about $1 \mathrm{~mm}$ apart. The split fiber hot-film probe has a $200 \mu \mathrm{m}$ platinum-plated nickel sensing element, which is about $1.25 \mathrm{~mm}$ long.

The sampling rates used in the measurements of HWA and HFA are $10 \mathrm{kHz}$ and $6 \mathrm{kHz}$, respectively. Determination of the flatness factor (i.e., Eq. (1)) follows the calculation process used in the study of Lee and Chang ${ }^{4}$ and is not repeated here for brevity. Uncertainties of both HWA and HFA measurements are estimated within $2.5 \%$ for both the mean and root-mean-squared fluctuating velocities. 
A two-dimensional PIV system is used for the instantaneous measurements of velocity field too. A continuous type, green-color laser (Elforlight HPG-6000, $532 \mathrm{~nm}$ wavelengths) with a cylindrical lens of $75 \mathrm{~mm}$ focal length generates a parallel, homogeneous light sheet with $50 \mathrm{~mm}$ in width and $3 \mathrm{~mm}$ in thickness and about $3.7 \mathrm{~W}$ powers in the operation of PIV measurement. A high speed video system (FASTCAM SA 5 type, Protron Co.) together with a set of a Nikon AIS $85 \mathrm{~mm} \mathrm{f} / 1.4$ lens and a Tokina $12 \mathrm{~mm}$ extension tube is used for image acquisition. The complementary- metal-oxidesemiconductor $(\mathrm{CMOS})$ sensor has $1024 \times 1024$ pixels and $20 \times 20 \mathrm{~mm}$ in real dimension. The tracers (seeding particles) are $\mathrm{SiO}_{2}$ with a nominal mean size $\left(\mathrm{d}_{50}\right)$ of $1.9 \mu \mathrm{m}$ and are seeded into the tested flow field by a cyclone particle generator in accordance with the seeding apparatus developed by Glass and Kennedy ${ }^{7}$.

The exposure time is dependent on the shutter speed of the camera rather than the pulse duration of the laser in use of a continuous laser. Considering a trade-off between acquiring enough intensity on the imaging sensor and reducing the blurred image of particles $^{8}$, the shutter speed is, thus, set to be $10^{-5} \mathrm{~s}$. The frame rate is equal to $10^{4} \mathrm{frame} / \mathrm{s}$ associated with the spatial resolution of $1024 \times 1024$ pixels. The magnification factor is set to be 0.191 in the measurement which leads to the spatial resolution in the image to be $0.105 \mathrm{~mm} /$ pixel. Turbulence statistics are computed using 10,000 pairs of frame to assure the statistically stationary results of turbulence. The cross correlation of image pairs is calculated with an ultimate interrogation window of $32 \times 32$ pixels using a grid refinement method and then performing window deformation. The grid refinement allows a higher spatial dynamic range ${ }^{9}$ while the window deformation reduces the error that is induced by velocity gradient in the flow field ${ }^{10}$. Note that the spatial resolution of the PIV depends on the employed size of the interrogation window. Deviations of mean velocities between the measurement of the PIV system and a cross-type hot-wire anemometer fall within 3\%. More detailed information on the PIV measurements in turbulent flow is referred to the study of $\mathrm{Li}$ et $\mathrm{al}^{11}$.

\subsection{Experimental conditions}

The experiments are separately conducted with the free stream (stream-wise) velocities of 6 and $15 \mathrm{~m} / \mathrm{s}$, which leads to the Reynolds numbers $\operatorname{Re}_{\mathrm{d}}$ (based on the diameter of cylinder) to be equal to $3.856 \times 10^{3}$ and $9.959 \times 10^{3}$, respectively. The free stream turbulent intensity is about $1 \%$. The measured stream-wise stations in this study are located at $\mathrm{x} / \mathrm{d}=5$. As justifying with the criteria repeated $\mathrm{in}^{1}$, the interests of investigation are located in the turbulent flow region. It was reported ${ }^{1}$ that a wake requires 50 to $100 \mathrm{~d}$ to reach its self-preserving condition. Obviously, the stations measured here are all located in the near-wake (or developing) region of the wake.

\section{Result and Discussion}

In general, intrusive probe techniques for the velocity measurements such as HWA and HFA are inherently simple and less expensive than the nonintrusive (mostly optical) methods. However, the presence of a physical probe can alter the local flow field around 
the probe. From this point of view, the size of $200 \mu \mathrm{m}$ sensing element in the split-fiber hot-film probe may cause larger disturbances in the flow measurements than the hot-wire probe with the sensing elements of $5 \mu \mathrm{m}$ in size does. Further, the larger size of sensing element of HFA leads to long response time (small sampling rate, $6 \mathrm{kHz}$ in this study) which disfavors the measurement of high-frequency (in other words, small-scale) part of turbulence as compared to the small size of sensing elements of HWA (10 kHz sampling rate). When applying for the two-dimensional flow measurements, the split-fiber probe can sense half-plane $\left( \pm 90^{\circ}\right)$ flow direction, while the X-array hot-wire probe can sense one-quadrant-plane $\left( \pm 45^{\circ}\right)$ flow direction only. From this point of view, split-fiber probe has wider measureable capability than the X-array hot-wire probe when applying to the measurements of multi-dimensional flow.

The nonintrusive diagnostic technique such as PIV is an indirect method since the data are made of the foreign particles (i.e., seedings) instead of the fluid flow itself. The uniformity of seeding and the lag of seeding motion from the fluid flow in the flow field particularly in the complicated flow are always the issues to be resolved. Overall, no simple and direct rule of thumb can be made that which diagnostic technique among the three employed instruments is the best choice in the present study.

Figure 2 presents that the planar distributions of mean stream-wise velocity (u), obtained by PIV measurement, at two Reynolds numbers. Due to the limit of the measurement capability of the PIV system, the measureable domain is $80 \mathrm{~mm}$ in length and $72 \mathrm{~mm}$ in width. The map shown in Fig. 2 is composed of two consecutive measured zones. In order to compare with the data obtained with point-wise measuring diagnostics, comparison of the sectional profiles of $\mathrm{U}, \mathrm{V}, \mathrm{u}_{\mathrm{rms}}$ ', $\mathrm{v}_{\mathrm{rms}}$ ', measured separately by HWA, HFA and PIV at the Reynolds numbers of $3.8 \times 10^{3}$ and $9.9 \times 10^{3}$, in the stream-wise station of $x / d=5$ are presented in Figs. 3-6, respectively. Note that the section of $x / d=5$ is located in the upstream region of the wakes where the flow direction near the centerline (i.e., $\mathrm{y}=0$ ) is far from the streamlined one. As mentioned previously, the $\mathrm{X}$-array hot-wire probe can sense one-quadrant-plane flow direction only. It implies that there might exist some places in this section where the X-array HWA is inapplicable. Justification of applicability of X-array HWA is made with the criteria as follows. Define the flow angle $\alpha$ as

$$
\alpha=\tan ^{-1}(u / v)
$$

where $\mathrm{u}$ and $\mathrm{v}$ are the instantaneous velocity of stream-wise and transverse components, respectively. If the percentage of the number of $\alpha$ data, in which their values fall within the angles between $-45^{\circ}$ and $45^{\circ}$, is greater than $95.45 \%$, which is two standard deviations of the mean in the normal distribution of instantaneous velocity, of the totally collected samples (100 k in this study), the applicability of X-array HWA at this position is valid; otherwise, the data obtained with X-array HWA will be deleted. Based on this justification process, it was found that X-array HWA cannot work properly in the inner cone region $(\mathrm{y} / \mathrm{d}<1)$ with $\mathrm{x} / \mathrm{d}=5$ in the case at $\mathrm{Re}_{\mathrm{d}}=3.8 \times 10^{3}$ (see Part (a) of Figs. 3-6). 
Nevertheless, X-array HWA can work properly in the whole section of $\mathrm{x} / \mathrm{d}=5$ at the relatively higher value of Reynolds number $\left(9.9 \times 10^{3}\right)$.

The comparisons made in Figs. 3 and 4 show that the mean velocity measurements ( $\mathrm{U}$ and $\mathrm{V}$ ) with three employed diagnostic techniques are in good agreement each other. In contrast, the measured $u_{\mathrm{rms}}$ ' and $\mathrm{v}_{\mathrm{rms}}$ ' using the three employed diagnostic techniques differ more significantly from one another (Figs. 5 and 6) as compared to the measurements of $U$ and V (Figs. 3 and 4). For the two intrusive techniques (HWA and HFA), the deviations of $u_{\mathrm{rms}}$ ' and $\mathrm{v}_{\mathrm{rms}}$ ' between them are remarkable in the inner shear turbulence regions (cf, Fig. 7) and are gradually alleviated moving outwardly to the free stream side. Nevertheless, the profiles of $u_{r m s}$ and $v_{r m s}$ measured with PIV deviate remarkably from the results measured either with HWA or with HFA in the whole section.
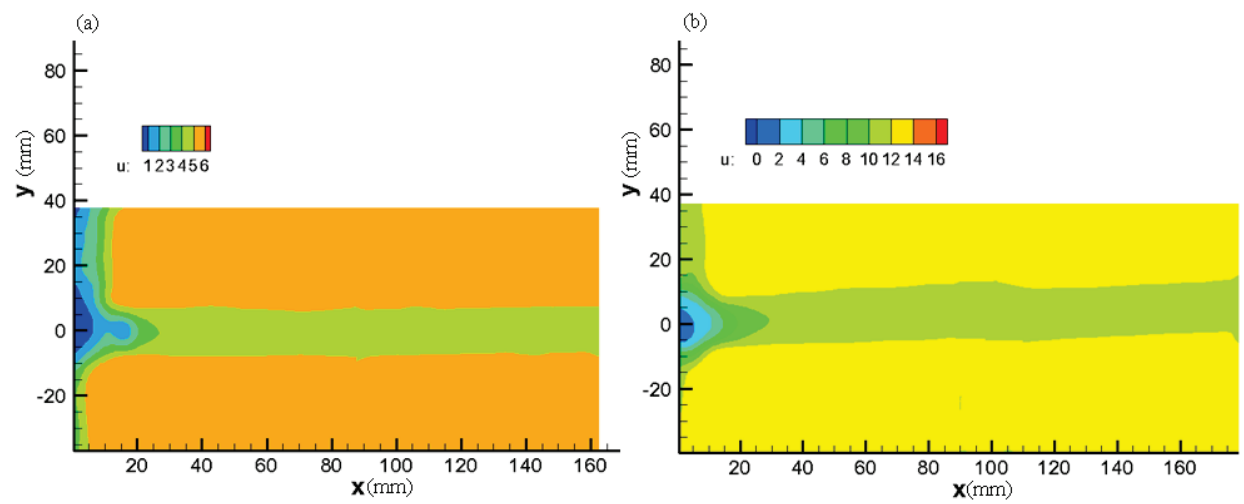

Fig. 2. Distributions of mean stream-wise velocity measured by PIV at Reynolds numbers of (a) $3.8 \times 10^{3}$ and (b) $9.9 \times 10^{3}$.
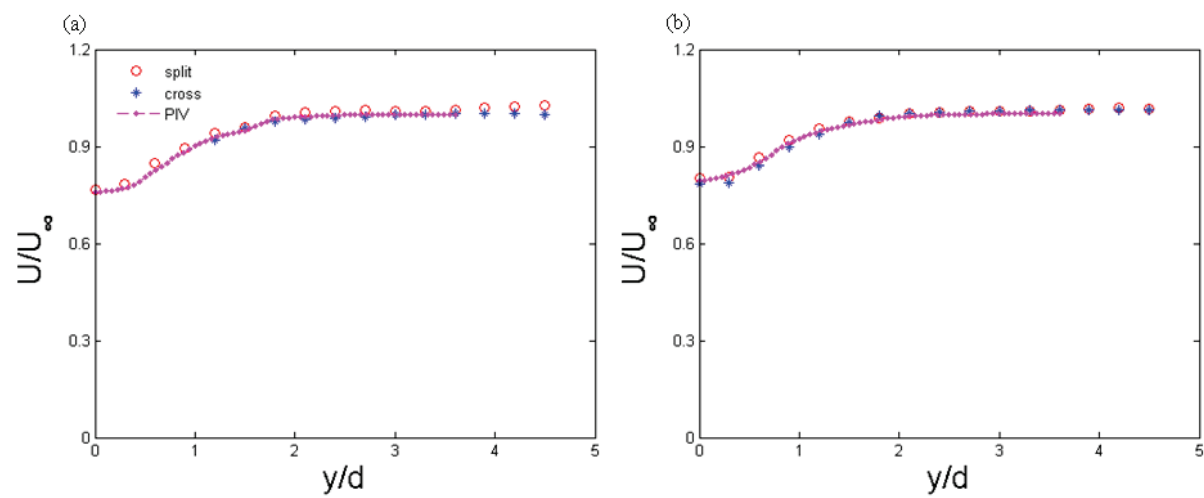

Fig. 3. Comparison of the mean stream-wise velocity profiles measured respectively by HWA, HFA and PIV in the stream-wise station of $\mathrm{x} / \mathrm{d}=5$ at Reynolds numbers of (a) $3.8 \times 10^{3}$ and (b) $9.9 \times 10^{3}$. 

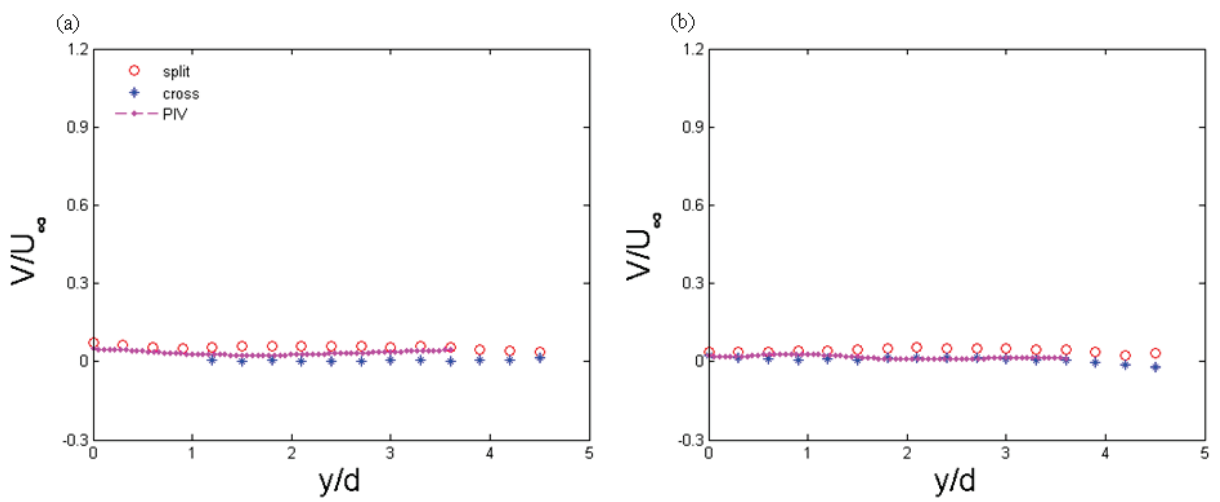

Fig. 4. Comparison of the mean transverse velocity profiles measured respectively by HFA, HFA and PIV in the transverse station of $\mathrm{x} / \mathrm{d}=5$ at Reynolds numbers of (a) $3.8 \times 10^{3}$ and (b) $9.9 \times 10^{3}$.
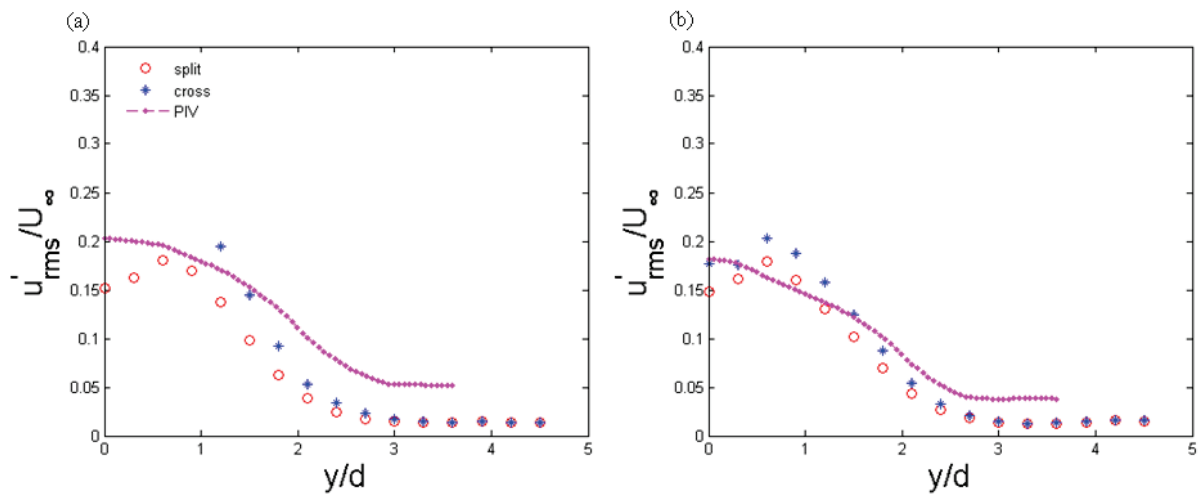

Fig. 5. Comparison of the root-mean-squared fluctuating stream-wise velocity profiles measured respectively by HFA, HFA and PIV in the transverse station of $\mathrm{x} / \mathrm{d}=5$ at Reynolds numbers of (a) $3.8 \times 10^{3}$ and (b) $9.9 \times 10^{3}$.
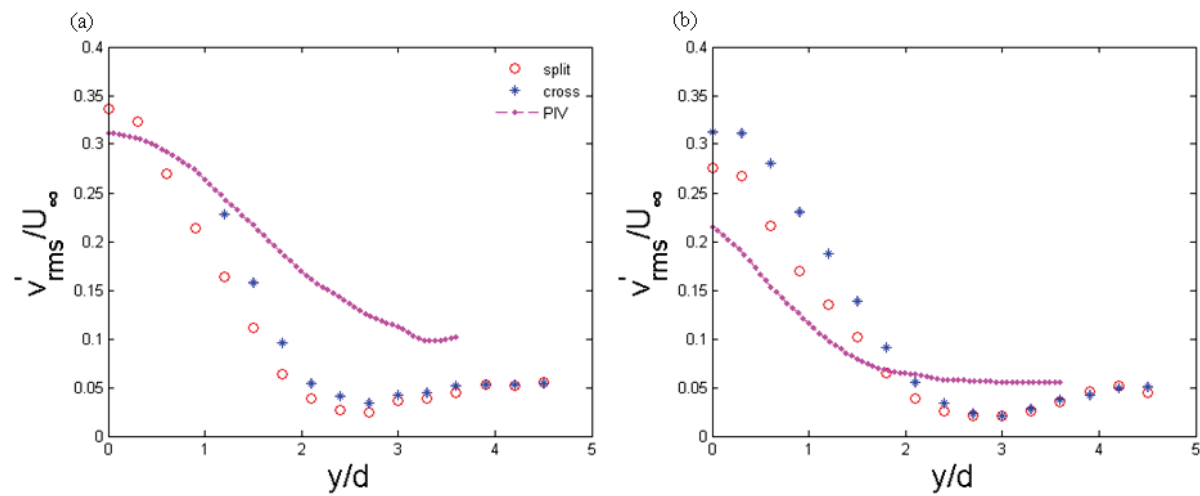

Fig. 6. Comparison of the root-mean-squared fluctuating transverse velocity profiles measured respectively by HFA, HFA and PIV in the transverse station of $\mathrm{x} / \mathrm{d}=5$ at Reynolds numbers of (a) $3.8 \times 10^{3}$ and (b) $9.9 \times 10^{3}$. 
Determination of the wake width in terms of the flatness factor requires the velocity information with high temporal resolution. As a result, the measured instantaneous stream-wise velocity using separately HWA and HFA at two investigated Reynolds numbers and at four stream-wise stations is used in the calculation of each corresponding sectional distribution of $F_{u}$. The peak positions of $F_{u}$ are recorded in Fig. 7 and can represent properly the wake width as claimed by Lee and $\mathrm{Chang}^{4}$.

Determinations of the wake width on the basis of shear-induced vorticity are also made with the PIV data of stream-wise velocity which provide good spatial resolution. Details of the calculation of shear-induced vorticity in two-dimensional Cartesian coordinate are referred to the appendix of Ref. 6 . The positions with zero value of the shear-induced vorticity are taken as the interface between the inner shear turbulence and the outer free stream and are recorded in Fig. 7.
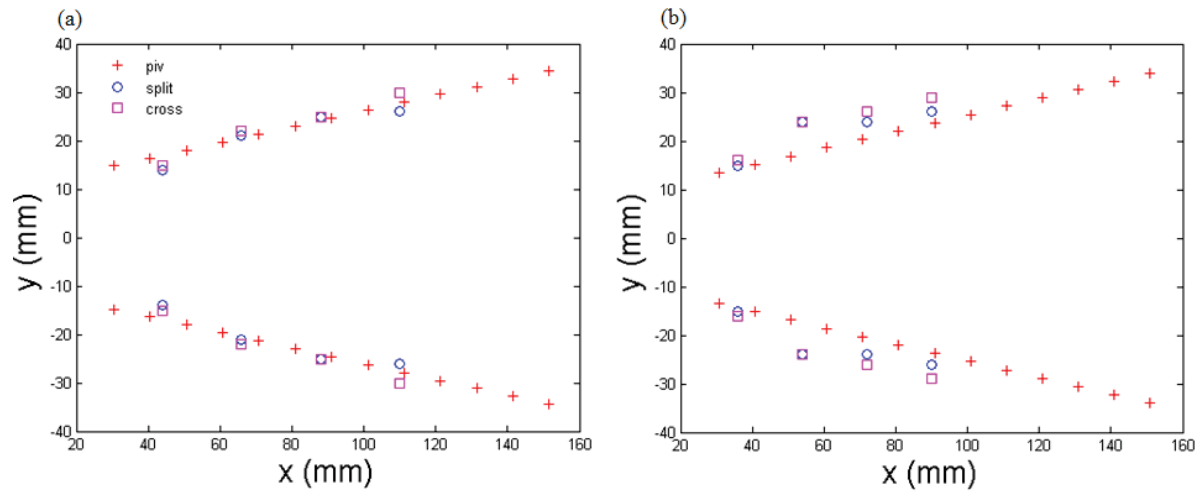

Fig.7. Comparison of the wakes width determined respectively on the bases of flatness factor and shearinduced vorticity at Reynolds numbers of (a) $3.8 \times 10^{3}$ and (b) $9.9 \times 10^{3}$.

Comparison made in Fig. 7 shows that the wake widths determined in terms of flatness factors with the data measured by either HWA or HFA are in good agreement to each other. The wakes widths determined in terms of shear-induced vorticity with the data measured by PIV are in good agreement with those defined in terms of flatness factor in the case of $\operatorname{Re}_{\mathrm{d}}=3.8 \times 10^{3}$, while are relatively narrower than those defined in terms of flatness factor in the case of $\operatorname{Re}_{\mathrm{d}}=9.9 \times 10^{3}$. Determination of the wake width on the basis of mean stream-wise velocity, $\mathrm{y}_{0.95}$, is also made with the information recorded in Fig. 3. For example, $\mathrm{y}_{0.95}=14.9$ and $13.0 \mathrm{~mm}$ at $\operatorname{Re}_{\mathrm{d}}=3.8 \times 10^{3}$ and $9.9 \times$ $10^{3}$, respectively, by using the data measured with HWA, at the stream-wise station of $\mathrm{x} / \mathrm{d}=5$ (i.e., $\mathrm{x}=50 \mathrm{~mm}$ ). Clearly, the wake width determined by $\mathrm{y}_{0.95}$ is narrower than those in terms of flatness factor and shear-induced vorticity. This fact corroborates with what were experimentally observed ${ }^{1-3}$ that the phenomenon of intermittency in the outer region of the wake width, defined by $l_{u}$, can be still observed. 


\section{Conclusions}

Turbulent flow measurements over a long, circular cylinder are performed with two intrusive diagnostics (i.e., HWA and HFA) as well as with one non-intrusive diagnostics (i.e., PIV). Comparison results show that all three employed diagnostic techniques yield similar measurements in the mean velocity (first order statistics) while somewhat deviated results in the root-mean-squared velocity (second order statistics), particularly for the PIV measurements. It is demonstrated that HFA possesses more capability than HWA in the flow measurements of wake flows. Wake width is determined in terms of either the flatness factor, which requires the velocity data with high temporal resolution, or shear-induced vorticity, which requires the velocity data with high spatial resolution. It is demonstrated in this study that the flow data obtained with the three employed diagnostic techniques are capable of yielding accurate determination of wake width.

\section{Acknowledgments}

This study was done with the financial support by National Science Council of R.O.C. under Grant No. NSC103-2923-E006-005-MY3.

\section{References}

1. A. A. Townsend, Australian Journal of Chemistry, 2(4), 451, (1949).

2. P. M. Bevilaqua and P. S. Lykoudis, AIAA Journal, 9(8), 1657, (1971).

3. M. M. Zdravkovich, Flow around Circular Cylinders : A Comprehensive Guide through Flow Phenomena, Experiments, Applications, Mathematical Models, and Computer Simulations, Chaps.1 and 5, (Oxford University Press, 1977)

4. K. H. Lee and K. C. Chang, Journal of Fluid Science and Technology, 9(3), (2014).

5. V. Kolar, International Journal of Heat and Fluid Flow, 28, 638, (2007).

6. K. C. Chang and C. T. Li, Journal of Fluid Science and Technology, 6, 662, (2011).

7. M. Glass and I. Kennedy, Combustion and Flame, 29, 333, (1977).

8. A. Boillot and A. K. Prasad, Experiments in Fluids, 21, 87, (1996).

9. J. Westerwell, Measurement Science and Technology, 8, 1379, (1997).

10. F. Scarano, Measurement Science and Technology, 13, R1, (2002).

11. C. T. Li, K. C. Chang, and M. R. Wang, Experimental Thermal and Fluid Science, 33, 527, (2009). 